\title{
LA PRUEBA DE ACCESO AL GRADO SUPERIOR DE CLARINETE: PAUTAS DE ACTUACIÓN METODOLÓGICA DESDE EL TERCER CICLO DE LAS EE.PP. DE MÚSICA
}

\author{
M. ${ }^{a}$ del Mar Bernabé Villodre \\ Universidad de Valencia \\ Francisco Joaquín Garres Pérez \\ Conservatorio Profesional de Música «Francisco Casanovas» de Torrevieja (Alicante)
}

\section{RESUMEN}

Al finalizar Enseñanzas Profesionales de Música, el estudiante realiza las pruebas de acceso a Grado Superior, consistentes en interpretar un repertorio orientativo, en repentizar un fragmento propuesto por el tribunal y en analizar una obra propuesta por el Departamento de Composición. Distintas entrevistas realizadas al profesorado de Grado Superior y al alumnado de último ciclo de EE.PP. mostraron las carencias académicas en distintos aspectos relacionados con estas pruebas y con la formación/aplicación didáctica. Orientadas a solucionar dicha situación, se aplicaron unas pautas de actuación metodológica para paliar las dificultades del alumnado del Tercer Ciclo de EE.PP. que se enfrenta a esta prueba de acceso a Grado Superior. Los resultados obtenidos en la muestra de alumnado mostraron cambios significativos respecto a los resultados previos a la aplicación, demostrando la necesidad de formar al alumnado desde propuestas constructivas del aprendizaje y obviando la tradicional pedagogía imitativa, característica de la tradición musical.

Palabras ClaVe: clarinete, repentización, interpretación, análisis, metodología, miedo escénico.

\section{THE ENTRANCE TEST TO THE HIGHER DEGREE OF CLARINET: GUIDELINES FOR METHODOLOGICAL ACTION SINCE THE THIRD CYCLE OF MUSIC EE.PP.}

\section{Abstract}

When finalizing Professional Music Education, the student takes the entrance exams to Higher Degree, consisting of interpreting an indicative repertoire, in repentizar a fragment proposed by the court and in analyzing a work proposed by the Department of Composition. Different interviews made to the Higher Grade teaching staff and the students of the last cycle of EE.PP., showed the academic deficiencies in different aspects related to these tests and to the didactic training/application. Oriented to solve this situation, some guidelines of methodological action aimed at alleviating the difficulties of the students of the Third Cycle of EE.PP. that faces this test of access to Higher Degree. The results obtained in the sample of students showed significant changes with respect to the results prior to the application, demonstrating the need to train students from constructive learning proposals and obviating the traditional imitative pedagogy, characteristic of the musical tradition. KEYWORDS: clarinet, repentization, interpretation, analysis, methodology, nerves.

DOI: http://doi.org/10.25145/j.qurricul.2018.31.003

Revista QuRRICULum, 31; junio 2018, pp. 57-75; ISSN: e-2530-8386 


\section{INTRODUCCIÓN}

La prueba de acceso a Grado Superior de Clarinete implica unas exigencias académicas que no terminan de corresponderse con los contenidos curriculares incluidos en la legislación vigente para el último ciclo de EE.PP. Esto supone que el docente deba trabajar con el alumnado obras de un nivel mayor al exigido para sexto curso, de cara a ser más competitivo en la prueba de acceso a estudios superiores de Música. De modo que los docentes de estas enseńanzas se encuentran en la misma disyuntiva que el profesorado de $2 .^{\circ}$ de Bachillerato, aunque con diferencias significativas: el profesorado de $2 .^{\circ}$ de Bachillerato trabaja los mismos contenidos de los que su alumnado se examina en la EBAU (Evaluación de Bachillerato para el Acceso a la Universidad), mientras que el profesorado de Clarinete del último curso de Tercer Ciclo de EE.PP. no trabaja todos los contenidos exigidos en las pruebas al Superior. Así que desde esta investigación se planteó seguir la lógica interna del profesorado de Bachillerato que prepara al alumnado desde los modelos de exámenes exigidos en la EBAU: se preparó al alumnado de sexto curso de EE.PP. siguiendo los modelos de exámenes de las pruebas de acceso realizadas en Conservatorios de Música Superiores, que se detallarán en epígrafes siguientes. Es necesario preparar al estudiante desde el punto de partida de las diferentes pruebas que componen el acceso al Grado Superior, no sólo para facilitarle el acceso, sino para garantizar la continuidad de una forma de trabajo constructivista y significativa durante los estudios superiores: se planteó cómo se estaba actuando con el alumnado, que cada vez estaba exigiendo más raciocinio, más comprensión, de las obras y menos imitación de las mismas por tanto, un aprendizaje con metodología constructivista y significativa alejado de la tradicional "pedagogía imitativa» característica de la enseñanza instrumental de los Conservatorios de Música.

El alumnado que cursa el último curso de Enseñanzas Profesionales de Música (EE.PP.) puede encontrarse con distintos problemas a la hora de realizar esta prueba de acceso a Grado Superior, tales como la falta de práctica en la repentización, problemas técnicos derivados del miedo escénico (excesiva tensión muscular que ahogue la columna de aire, excesiva salivación...) o problemas a la hora de interpretar las características estructurales y formales de las distintas piezas musicales. En la figura 1, se pueden observar las opiniones de los 23 estudiantes aspirantes al Grado Superior de Clarinete que fueron entrevistados para conocer sus opiniones ante la prueba de acceso, las principales carencias y problemas con los que se encontraban ante la misma. Estos datos, obtenidos de la totalidad de los clarinetistas aspirantes a lo largo de tres cursos académicos (2013/2014, 2014/2015 y 2015/2016) en los Conservatorios Superiores de Murcia y de Alicante, permitieron conocer el contexto en el que se pretendía desarrollar la propuesta que se incluye en este artículo.

Para hacer frente a estos problemas, que también fueron detectados por el profesorado de Profesional y Superior entrevistados (figura 2 y tablas II y III), se pusieron en práctica diferentes pautas de actuación para intentar paliar las dificultades surgidas de las exigencias específicas de esta prueba y facilitar el acceso a estudios superiores de Música. La estructura organizativa de dichas pautas vino determinada por un análisis previo de la legislación referente a las pruebas de acceso 


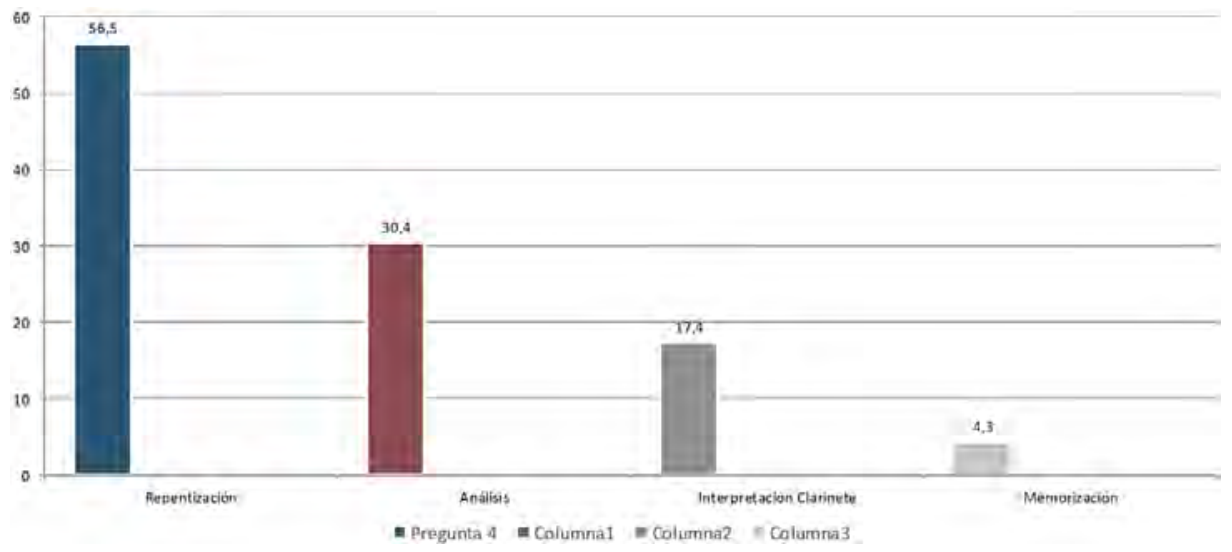

Fuente: Elaboración propia.

Figura 1. Partes más problemáticas de la prueba de acceso a Grado Superior de Clarinete.

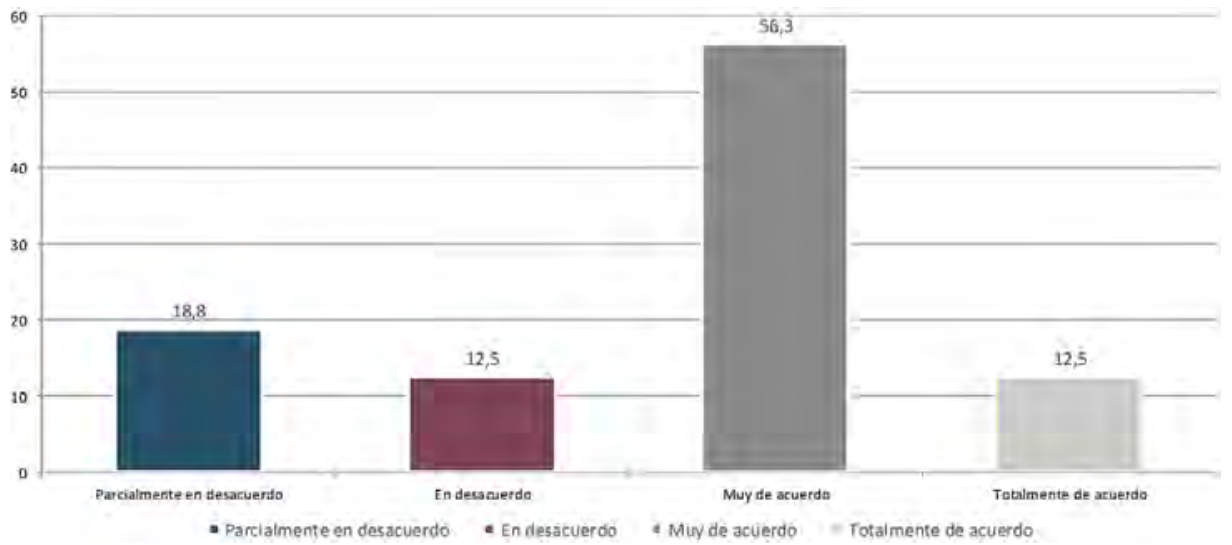

Fuente: Elaboración propia.

Figura 2. Consideración de la existencia de problemas metodológicos en EE.PP. por parte del profesorado de Superior y de Profesional.

a Grado Superior (Plan de 1966, LOGSE y LOE), que mostró cómo el profesorado que realizaba estas pruebas seguía planteando obras de referencia acordes con planes de estudios antiguos que ya no tenían razón de ser ante la evolución curricular del plan de estudios actual (LOE).

Las obras exigidas en la prueba de acceso de Clarinete tienen un nivel técnico y musical que supera con creces lo planteado en la legislación educativa de EE.PP. Esto supone que, a nivel madurativo, tanto personal como musical, el alumnado necesita unas herramientas para hacerle frente; lo que implica que el docente de Cla- 
rinete enseñe a analizar las obras que se interpretarán, tanto desde las características estilísticas como estructurales relevantes para mejorar la interpretación, y evite la tradicional imitación o audición de un disco compacto para imitarla igualmente. En este sentido, Bautista y Pérez-Echeverría (2008) consideran que es muy importante que el alumnado sepa procesar los distintos elementos analíticos de una partitura. En epígrafes posteriores, se detallará detenidamente el enfoque de esta práctica analítica específica.

A la problemática sobre los contenidos a trabajar para poder superar una prueba de acceso, se unió el hecho de que en la especialidad de Clarinete no se contaba con métodos didácticos específicos para trabajar en el aula. Cierto que había muchos libros cuyo título era Método para Clarinete (por ejemplo, el de Antonio Romero), pero no se contaba con investigaciones, ni documentos centrados en el enfoque del trabajo didáctico realizado con ellos; mientras que en Lenguaje Musical sí se contaba con una metodología Orff, Dalcroze, etc., o en Violín, una metodología Suzuki. La formación didáctica del profesorado de Clarinete no ha ocupado demasiadas investigaciones cualitativas y/o cuantitativas en España (Bernabé, 2013; Borrás, 2015, por ejemplo), siendo más abundantes las musicológicas (Abella, 2015; Álvarez, 2014), centradas en el análisis del repertorio clarinetístico. Esto podría explicarse por dos motivos: la tradición de lo que podría denominarse una pedagogía imitativa está muy arraigada en el mundo de las especialidades instrumentales de los Conservatorios de Música; y, la formación didáctica que recibe el clarinetista no suele ser de carácter troncal u obligatorio, sino más bien optativo o de libre configuración (Bernabé y Torres, 2017). Y esto último sólo ha venido sucediendo desde la aplicación del Plan LOGSE en los estudios de Grado Superior de Música, porque en el Plan de 1966 no se contaba con un currículo formativo tan amplio como el propuesto durante la LOGSE, que se perfeccionaría con la LOE.

Todo esto llevó a considerar la relevancia de investigar en la didáctica instrumental con la intención de crear todo un campo específico, que contribuyese a la mejora de la disciplina de la didáctica del Clarinete, al tiempo que a mejorar los resultados en las pruebas de acceso, gracias al desarrollo de una didáctica constructiva y significativa como se propone en este artículo.

\section{OBJETIVOS}

Las Enseñanzas Artísticas, principalmente las de carácter instrumental, precisan demostrar que su proceso de enseñanza/aprendizaje no se reduce a un proceso imitativo, sino que hay todo un entramado complejo que dará como resultado una interpretación musical de calidad. Esta inexistencia de una sistematización metodológica en el proceso de enseñanza/aprendizaje del Clarinete en los Conservatorios Profesionales de Música llevó a considerar hasta qué punto se podía decir que el alumnado contaba con la preparación adecuada para superar dicha prueba. De modo que esta falta de metodología específica del Clarinete llevó a plantear como objetivo principal la sistematización de una metodología para el último ciclo, que facilitase una exitosa prueba de acceso al Grado Superior, además de mejorar 
la interpretación del alumnado del Tercer Ciclo de EE.PP. que iba a presentarse a la prueba de acceso. Es decir, que se pretendió concretar unas pautas didácticas específicas para el último ciclo de EE.PP., vertebradas en torno a las exigencias de la prueba de acceso al Grado Superior de Clarinete, pero que al mismo tiempo respondiesen a las exigencias concretas del Tercer Ciclo de EE.PP.

A raíz de esta problemática detectada, surgieron tres objetivos específicos:

- Plantear pautas para repentizar.

- Estructurar el trabajo del proceso de memorización de las obras musicales.

- Organizar el trabajo del análisis del repertorio clarinetístico.

\section{METODOLOGÍA}

La organización y la estructuración didácticas del último ciclo de EE.PP. de acuerdo con las exigencias de la prueba de acceso al Grado Superior garantizarán que se termine de asentar el paralelismo entre el acceso a estos estudios musicales y el acceso a la Universidad. Igual que sucede en $2 .^{\circ}$ curso de Bachillerato, el último ciclo de estas Enseñanzas Profesionales debería limitarse a trabajar los contenidos específicos del mismo, y no el nivel exigido en las pruebas de acceso a Grado Superior, ya que el estudiante preuniversitario no se examina de Didáctica de la Expresión Corporal porque quiera estudiar Magisterio Mención Educación Física. Aunque, más allá de esta consideración, el objetivo principal planteado se muestra como una realidad legalmente aceptada y posible, al no alejarse de lo establecido en la legislación vigente; pero, al mismo tiempo, puede convertirse en una metodología didáctica que dote de sistematización a una disciplina basada demasiado tiempo en la imitación.

\subsection{Muestra}

Se trabajó con la población (docentes y alumnado) de los Conservatorios Profesionales de Música de la comarca de la Vega Baja de la Comunidad Valenciana, de las ciudades de Alicante y Murcia, que reciben mucho alumnado procedente de esta comarca que, por proximidad geográfica, se vincula más con la Región de Murcia para cursar estudios musicales que con Valencia.

De esta población, para la realización del proceso de sistematización metodológica se seleccionó una muestra de alumnado que cursaba estudios de último ciclo de Enseñanzas Profesionales de Clarinete en el Conservatorio Profesional de Música «Francisco Casanovas» de Torrevieja (Alicante). Aunque al finalizar la investigación se realizaron experiencias de aplicación de la metodología propuesta en los distintos centros musicales de la Vega Baja, gracias al intercambio entre Conservatorios de Música promovidos tradicionalmente por la amistad del profesorado. Gracias precisamente a ello, se contó con la participación de profesorado de Clarinete de los distintos Conservatorios que se enumeran más abajo, así como de sus especialistas de Composición y de miedo escénico; puesto que una búsqueda bibliográfica del 
estadio previo a la realización de esta investigación demostró que no se contaba con fuentes bibliográficas relacionadas y había que recurrir a las fuentes directas, en este caso, el profesorado.

A continuación, se enumeran centros y número de profesorado implicado en la recogida de datos cualitativos para fundamentar esta investigación (tabla I). Además, se obtuvieron importantes datos para fundamentar la necesidad de investigar en una mejora de los procedimientos didácticos realizados en el aula de Clarinete. Esta muestra de alumnado que había realizado las pruebas de acceso representó a la totalidad de los aspirantes durante tres cursos académicos (2013/2014, 2014/2015 y 2015/2016), en este caso, el número ascendió a 23 personas.

\begin{tabular}{ccc}
\hline TABLA I. RELACIÓN PROFESORADO-CENTRO-ESPECIALIDAD \\
\hline CONSERVATORIO & $\begin{array}{c}\text { NúMERO DE } \\
\text { PROFESORADO }\end{array}$ & ESPECIALIDAD \\
\hline $\begin{array}{c}\text { Conservatorio Superior } \\
\text { de Música de Alicante }\end{array}$ & 3 & Clarinete \\
\hline $\begin{array}{c}\text { Conservatorio Profesional } \\
\text { «Francisco Casanovas» de Torrevieja }\end{array}$ & 1 & Clarinete \\
\cline { 2 - 3 } & 1 & Composición \\
\hline $\begin{array}{c}\text { Conservatorio Superior de Música } \\
\text { «Manuel Massotti Littel» de Murcia }\end{array}$ & 2 & Clarinete \\
\cline { 2 - 3 } & 1 & Composición \\
\hline $\begin{array}{c}\text { Conservatorio Profesional } \\
\text { de Música de Murcia }\end{array}$ & 1 & Clarinete relajación \\
\hline $\begin{array}{c}\text { Conservatorio Profesional } \\
\text { de Música de Catral }\end{array}$ & 1 & Miedo escénico \\
\hline $\begin{array}{c}\text { Conservatorio Profesional de Música } \\
\text { de Pilar de la Horadada }\end{array}$ & 1 & Clarinete \\
\hline $\begin{array}{c}\text { Conservatorio Profesional } \\
\text { de Música de Orihuela }\end{array}$ & 1 & Clarinete \\
\hline $\begin{array}{c}\text { Conservatorio Profesional } \\
\text { de Música de Almoradí }\end{array}$ & 3 & Clarinete \\
\hline
\end{tabular}

Fuente: elaboración propia a partir de los datos obtenidos.

Se contó con la colaboración de tres especialistas en miedo escénico de centros localizados fuera de la ciudad de Murcia y de la comarca de la Vega Baja, zona donde se localizó esta investigación.

En cuanto al alumnado de Clarinete del Conservatorio de Torrevieja con quienes se desarrolló esta investigación, fueron ocho y representaba a la totalidad de la población que estudiaba Clarinete en el Conservatorio Profesional de Música «Francisco Casanovas» de Torrevieja. 


\subsection{INSTRUMENTOS}

La investigación se dividió en tres momentos caracterizados por la presencia de distintos instrumentos de recogida de datos, que fueron validados por expertos. Para la justificación de la línea de investigación propuesta, centrada en la necesidad de crear una didáctica del Clarinete en los Conservatorios de Música, se contó con diferentes cuestionarios (tabla II) y entrevistas (tabla III) destinados a recoger datos cuantitativos y cualitativos de las fuentes más directas. Esto se debió a la práctica inexistencia de referencias bibliográficas relacionadas, lo que terminó de justificar esta investigación. De modo que, para la cumplimentación del cuestionario incluido en la tabla II, se recurrió a un modelo escala de Likert con las siguientes posibilidades de respuesta: 0- Totalmente en desacuerdo; 1- Parcialmente en desacuerdo; 2- En desacuerdo; 3- Parcialmente de acuerdo; 4- Muy de acuerdo; y 5 - Totalmente de acuerdo. En esta tabla II, también puede observarse una división que marca las preguntas referentes a las pruebas de acceso al Grado Superior de Clarinete.

\section{TABLA II. CUESTIONARIO REALIZADO POR EL PROFESORADO DE EE.PP. Y GRADO SUPERIOR DE CLARINETE}

1. Estoy de acuerdo con la programación de clarinete de EE.PP. establecida por la legislación...

2. Es necesario realizar algunos cambios en el currículo de clarinete de EE.PP.

3. Se deben cambiar las obras en el último ciclo de EE.PP.

4. Ha bajado el nivel en las pruebas de acceso a Enseñanzas Profesionales.

5. El alumnado tiene muchos problemas con el Lenguaje Musical.

6. El alumnado llega con problemas técnicos con el clarinete en el acceso a EE.PP.

7. Las obras de clarinete propuestas para acceso a E.S. no se ajustan a los contenidos trabajados en EE.PP.

8. La bibliografía del último ciclo de EE.PP. es correcta.

9. Debemos ofertar obras de clarinete con menor nivel en EE.PP. (último ciclo).

10. Estas obras tan difíciles se deben realizar en el Superior.

11. El alumnado nunca finaliza la programación del último ciclo de EE.PP. debido a su dificultad.

12. Existen problemas con la metodología a partir del 2. ${ }^{\circ}$ ciclo de EE.PP.

13. Hay obras en el tercer ciclo de EE.PP. que el alumnado no puede interpretar.

14. No debemos colocar algunas obras tan difíciles de interpretar.

15. El número de pruebas es suficiente.

16. Las pruebas son de nivel muy alto.

17. El examen de Repentización es el correcto.

18. Los alumnos tienen muchos problemas en la interpretación y lectura de la repentización.

19. Los problemas de Lenguaje Musical afectan negativamente en la prueba de repentización.

20. Las obras propuestas para clarinete son las idóneas.

21. Debemos bajar el nivel de las obras.

22. Hay obras en el último ciclo de EE.PP. que se estudian en el Superior. 
23. Las obras del Superior no deben estudiarse en las EE.PP., al igual que en la EBAU no se exigen conocimientos de medicina, etc.

24. Se deben seleccionar mejor las obras para la prueba al Superior.

25. Tienen muchos problemas de lectura musical a primera vista.

26. Deben mejorar mucho en la prueba de análisis.

27. El alumnado tiene muchos problemas en las tres partes de la prueba de acceso.

Fuente: elaboración propia.

En la tabla III, puede observarse una división que señala las preguntas referentes a Enseñanzas Profesionales de aquellas relacionadas con la prueba de acceso al Grado Superior:

\section{TABLA III. PREGUNTAS REALIZADAS AL PROFESORADO DE CLARINETE DE PROFESIONAL Y SUPERIOR}

1. Igual que sucede en la PAU, en el 3 er $^{\text {er }}$ Ciclo de E.P. ¿¿deben trabajarse obras diferentes a las del Superior?

2. Deben regularizarse mejor los libros de textos musicales y metodología musical (didáctica específica del Clarinete) en las E.P., para afrontar correctamente la prueba al Superior.

3. Deben establecerse obras con menor dificultad técnica, acordes con los contenidos establecidos en la legislación vigente.

4. La técnica del alumnado es deficiente para poder superar el nivel exigido en el último ciclo.

5. Propone algunos cambios (contenidos, metodología, obras...) para el tercer ciclo de E.P.

6. Plantea trabajar otros aspectos como, por ejemplo, la memorización y la concentración.

7. Debe estudiar el alumnado las obras que posteriormente interpretan en el Superior, al igual que en Bachillerato y en la Universidad.

8. Debe establecerse una mejor sistematización metodológica/didáctica en el tercer ciclo de E.P., para afrontar de forma satisfactoria la prueba de acceso al Superior.

9. Se debe trabajar más en la lectura a primera vista o repentización.

10. Los métodos para clarinete son suficientes para afrontar el tercer ciclo de E.P. y prueba al Superior.

11. Propone cambios para la prueba al Superior.

12. Considera que la prueba de análisis exige un nivel mayor al conseguido por el alumnado al finalizar E.P.

13. El alumnado afronta correctamente el nivel exigido en $1 .^{\circ}$ de Superior.

14. Qué problemas observa en la memorización de las obras.

15. Qué problemas observa en la repentización.

16. Las obras que proponen al alumnado son las adecuadas al nivel de donde proceden.

17. Las obras propuestas por el Seminario/Departamento para el acceso al Superior son las adecuadas.

18. Técnicamente cómo ve al alumnado que se presenta.

19. En qué exámenes observa más carencias al alumnado.

20. Está de acuerdo con los diferentes exámenes que se realizan en la prueba de acceso a Grado Superior.

Fuente: elaboración propia. 
Orientada a la propuesta que se realizaría en el citado Conservatorio de Música, para organizarla de acuerdo con las necesidades del alumnado de cara a la prueba de acceso al Conservatorio Superior de Música, se realizaron una entrevista (tabla Iv) y un cuestionario (tabla v) durante el primer curso de Grado Superior de Clarinete del alumnado aspirante en cursos anteriores. De esta forma, se complementaba la información necesaria para la organización de la propuesta metodológica desarrollada:

\section{TABLA IV. ENTREVISTA AL ALUMNADO ASPIRANTE AL GRADO SUPERIOR DE MÚSICA}

1. ¿Has finalizado los estudios de Enseñanzas Profesionales?

2. ¿Tienes todos los requisitos exigidos (EE.PP. Bachillerato) para optar a la prueba al Superior?

3. ¿Te encuentras preparado para las pruebas al Superior?

4. ¿Con cuál de los tres exámenes tienes más problemas?

5. ¿Estás satisfecho con lo aprendido en las EE.PP.?

6. ¿Qué tal llevas las obras propuestas para la prueba instrumental y qué dificultad tiene?

7. ¿Qué aspectos necesitas trabajar más? Por ejemplo: memorización de la obra, concentración, análisis musical, lectura a primera vista, etc.

8. ¿Cómo calificas los exámenes propuestos para la prueba al Superior?

9. ¿ ¿Tienes la información necesaria para afrontar correctamente la prueba al Superior?

10. ¿En qué parte de la prueba tienes más carencias?

Fuente: elaboración propia.

\section{TABLA V. CUESTIONARIO PARA EL ALUMNADO QUE CURSABA} PRIMER CURSO DE GRADO SUPERIOR DE CLARINETE

1. ¿Cómo has afrontado el comienzo del curso?

2. ¿Encuentras muchas diferencias entre el último ciclo de EE.PP. y el Grado Superior?

3. ¿Consideras que te encuentras preparado para afrontar el $1 .{ }^{\text {er }}$ Curso de Grado Superior?

4. ¿Qué aspectos clarinetísticos estás trabajando más o diferentes a los de Enseñanzas Profesionales?

5. ¿Cómo afrontas la asignatura de Análisis? ¿Encuentras mucha diferencia respecto al último ciclo de EE.PP.?

6. ¿El primer curso ha superado tus expectativas?

7. Las obras de clarinete que interpretas actualmente, ¿las habías interpretado ya en EE.PP.?

8. ¿Qué asignaturas abordas mejor y en cuáles tienes problemas?

9. ¿Qué aspectos cambiarías o trabajarías más en EE.PP., si tuvieras que presentarte otra vez a la prueba al Superior?

10. En caso de volver a presentarte a la prueba al Superior, ¿elegirías otra vez Clarinete o bien Pedagogía/ Musicología?

Fuente: elaboración propia. 
Así, se organizaron estos datos con la información recabada del profesorado y se procedió al segundo momento de recogida de datos, que fue el de aplicación de la propuesta metodológica: para ello, se pasó un test previo (tabla vi) para comprobar el estado del alumnado y que fue pasado tras la intervención con la intención de observar su evolución. El último momento se centró en la organización, clasificación y comparación de los resultados obtenidos de los cuestionarios anteriores, mediante el programa informático SPSS.

\begin{tabular}{ll} 
& TABLA VI. CUESTIONARIO DEL ALUMNADO PARTICIPANTE EN LA EXPERIENCIA \\
\hline 1. & ¿Realizas algún ejercicio de respiración con el clarinete o sin el clarinete? \\
\hline 2. & ¿Calientas con ejercicios de intervalos, arpegios o escala cromática? \\
\hline 3. & Al interpretar una obra en audiciones, ¿qué es lo que más te preocupa? \\
\hline 4. & ¿Cómo trabajas las obras en casa? ¿Las analizas y estudias el estilo musical? \\
\hline 5. & ¿Qué problemas tienes en la lectura a primera vista? \\
\hline 6. & ¿Cómo memorizas una obra musical? \\
\hline
\end{tabular}

Fuente: elaboración propia.

\subsection{Procedimiento}

La prueba de acceso para especialidades instrumentales consiste en tres exámenes eliminatorios: repentización o lectura a primera vista de una melodía, análisis de una obra no clarinetística, interpretación con el clarinete (acompañado al piano y una solo) de tres obras de diferentes estilos musicales, siendo obligatoria la memorización de una. De acuerdo con esto, el profesorado de último ciclo de Profesional está «obligado» a orientar éste para garantizar que su alumnado vaya suficientemente preparado a dichas pruebas, en detrimento de otros aspectos contenidos en el currículo del ciclo.

De acuerdo con esta consideración, se realizaron unas propuestas metodológicas para el último ciclo de EE.PP. orientadas a superar la prueba de acceso al Grado Superior. Éstas fueron desarrolladas durante el curso académico 2016/2017, en el Conservatorio Profesional de Música de Torrevieja. Dichos planteamientos pretendieron establecer un nivel interpretativo óptimo que ayudase al estudiante a ser consciente de su propia interpretación y de sus posibilidades musicales, además de que se asentasen las bases de una sistematización metodológica para el último ciclo de EE.PP. que estuviese en armonía con las exigencias del acceso a Grado Superior.

En primer lugar, para trabajar las obras del repertorio se consideró que debía partirse de los parámetros del análisis musical como modo fundamental de aportar al alumnado los criterios interpretativos justificados para guiar su interpretación (Bautista y Pérez-Echeverría, 2008). El hecho de razonar el fraseo a nivel armónico, formal e histórico-estilístico los capacitaría para comprender la interpretación y, al mismo tiempo, para dotar de mayor sentido cualquier obra repentizada. La repentización formaba parte de las pruebas del examen de acceso, de manera que 
el estudiantado también tenía que saber enfrentarse a una obra a primera vista; $y$, para ello, no bastaba sólo con la suficiente agilidad técnica, sino que el sentimiento interpretativo derivado del conocimiento de un estilo musical mejoraría la interpretación del músico.

El análisis se trabajó desde la partitura de clarinete contrastada con la del piano, para encontrar los puntos armónicos clave que llevarían a la localización exacta del fraseo, la estructura y la expresividad consiguiente, derivada del uso cadencial. A todo esto, se sumó el hecho de que cuanto más se ahondaba en los elementos característicos del análisis musical, más fácil resultaba memorizar la obra.

En segundo lugar, se trabajó la memoria musical, muy relacionada con el proceso de estudio analítico de la partitura, como se comentó anteriormente. Para este trabajo, se partió de los beneficios, de las facilidades, de ese estudio analítico de la partitura clarinete-piano; a lo que se sumó la repetición de las frases y de los períodos con el acompañamiento del piano, lo que facilitaba el proceso de memorización del clarinetista.

La repentización propuesta por los tribunales de Alicante y Murcia, normalmente, es de corte contemporáneo y compuesta expresamente para la prueba de acceso por el profesorado del Departamento de Composición de cada uno de esos centros. La música contemporánea implica unos parámetros analíticos muy diferentes de los que entran en juego en una partitura clásica o incluso una romántica; situación que llevó a trabajar pequeños fragmentos de obras contemporáneas que aproximasen al estudiante a las peculiaridades del repertorio actual. En este sentido, los solos de obras orquestales contemporáneas ofrecían un interesante campo de trabajo para la interpretación de las obras contemporáneas; además de que, a nivel técnico, también ofrecían similar grado de dificultad.

En cuanto a la interpretación de las obras, algunas debían interpretarse con pianista acompañante, otras sin pianista, algunas de memoria y otras con partitura. Ante estas exigencias de la prueba de acceso, se añadieron algunas pautas de trabajo en el aula del estudiante con otros estudiantes y en el auditorio solo y con otros estudiantes de público como forma de facilitar el autocontrol ante los nervios de la interpretación en público, que podían terminar derivando en ese miedo escénico que ha llegado a truncar algunas carreras interpretativas.

El estudiante sólo podía interpretar las obras una vez que las había analizado desde todos los parámetros posibles, desde que considerase que controlaba la capacidad respiratoria y la técnica específica, desde que se viese capaz de interpretar controlando la columna de aire y su propio cuerpo ante la tensión que pudiese provocar la interpretación en el aula / en el escenario / ante un tribunal. Para lograr esto, en el aula de Clarinete se propuso la realización de pequeñas audiciones que contribuyesen a aumentar la seguridad del estudiante ante su propia interpretación, aunque lo que se mostró como más eficaz fue el traslado de la clase al escenario (sin público) como forma de familiarizarse con el entorno para calmar el nerviosismo del estudiante ante el acto en sí.

La organización del tiempo de la clase de Clarinete también debía responder a un esquema concreto para dedicar el tiempo adecuado a cada uno de los elementos a trabajar. Toda metodología necesita de una rigurosa sistematización temporal que 


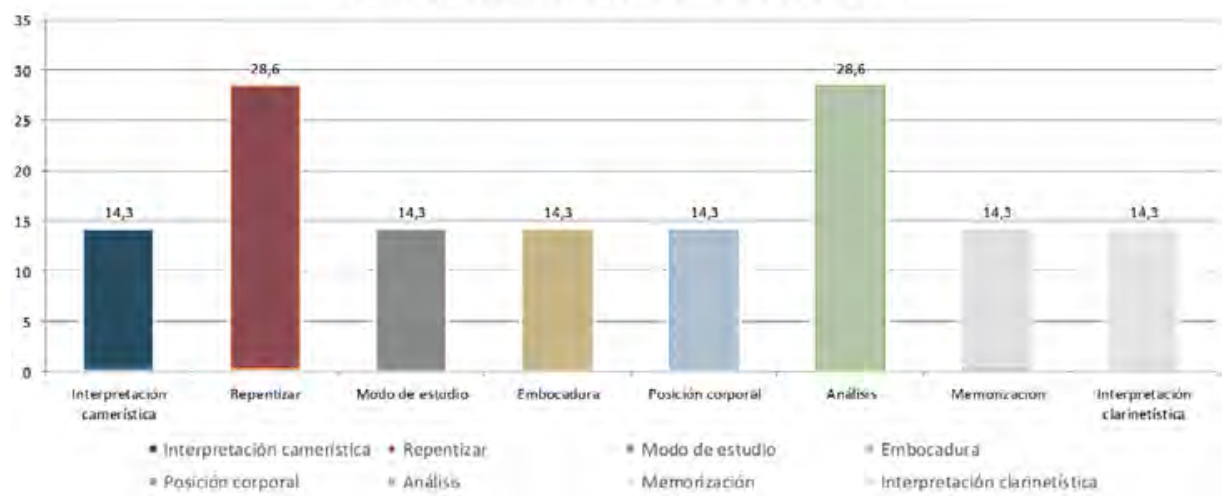

Fuente: elaboración propia.

Figura 3. Cambios señalados por el alumnado de $1 .^{\circ}$ de Grado Superior de Clarinete.

lleve a considerar la flexibilidad necesaria para momentos puntuales de trabajo en problemas o carencias detectados. Así, primero, se iniciaba la clase con un trabajo respiratorio y muscular para calentar cuerpo y clarinete, al tiempo que para centrar la mente del estudiante; después, se pasaba al trabajo de la técnica, imprescindible para la interpretación y la repentización, al tiempo que para dotar de seguridad al músico ante su interpretación en público; posteriormente, se trabajaba el proceso de memorización y repentización mediante pequeñas obras melódicas de la dificultad exigida al curso correspondiente; $y$, para finalizar, se trabajaron las obras específicas de las pruebas de acceso con y sin el pianista acompańante, teniendo en cuenta que el trabajo se iniciaba con el análisis del repertorio para posibilitar una interpretación significativa.

\section{RESULTADOS}

Los resultados obtenidos en las entrevistas al alumnado de $1 .^{\circ}$ de Grado $\mathrm{Su}$ perior de los Conservatorios Superiores de Música de Murcia y de Alicante (figura 3 y tabla v) permitieron justificar la propuesta metodológica desarrollada y, por tanto, los resultados del alumnado que se detallan más abajo (figuras 6 y 7 ).

De las preguntas contempladas en la tabla v, también se obtuvieron datos referentes a las deficiencias de formación (figura 4) que el alumnado de Clarinete había detectado al entrar en el primer curso de Grado Superior, y que consideraban no deberían haber tenido de cara a la formación como especialistas en Clarinete. Estos datos fueron importantes para poder realizar la propuesta ya comentada con mayor rigurosidad, atendiendo a las necesidades del alumnado de Clarinete, que 


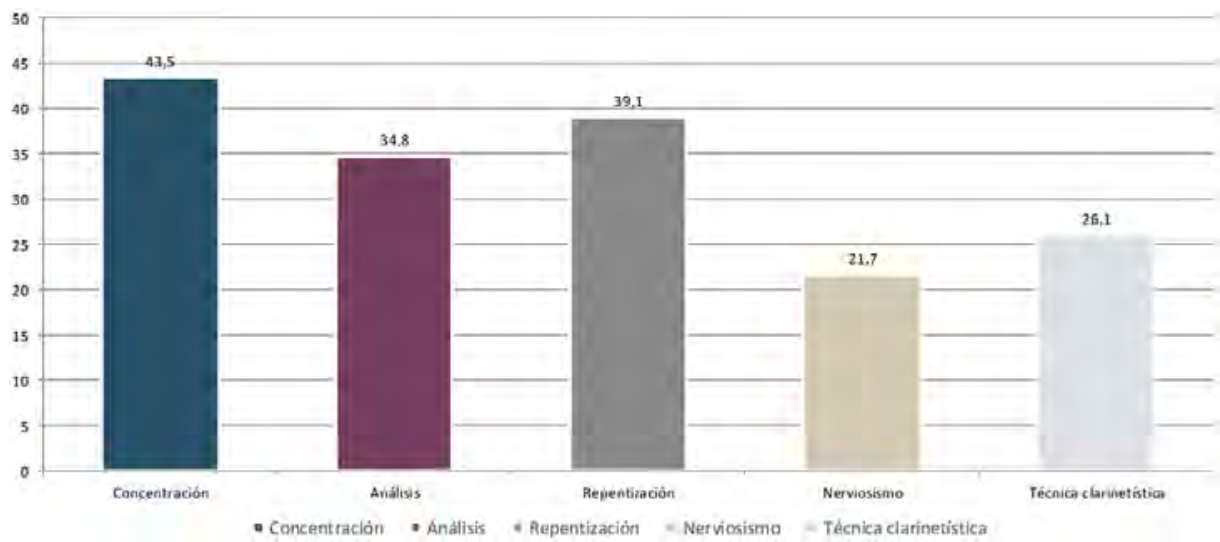

Fuente: elaboración propia.

Figura 4. Deficiencias formativas autodetectadas por el alumnado de $1 .^{\circ}$ de Grado Superior.

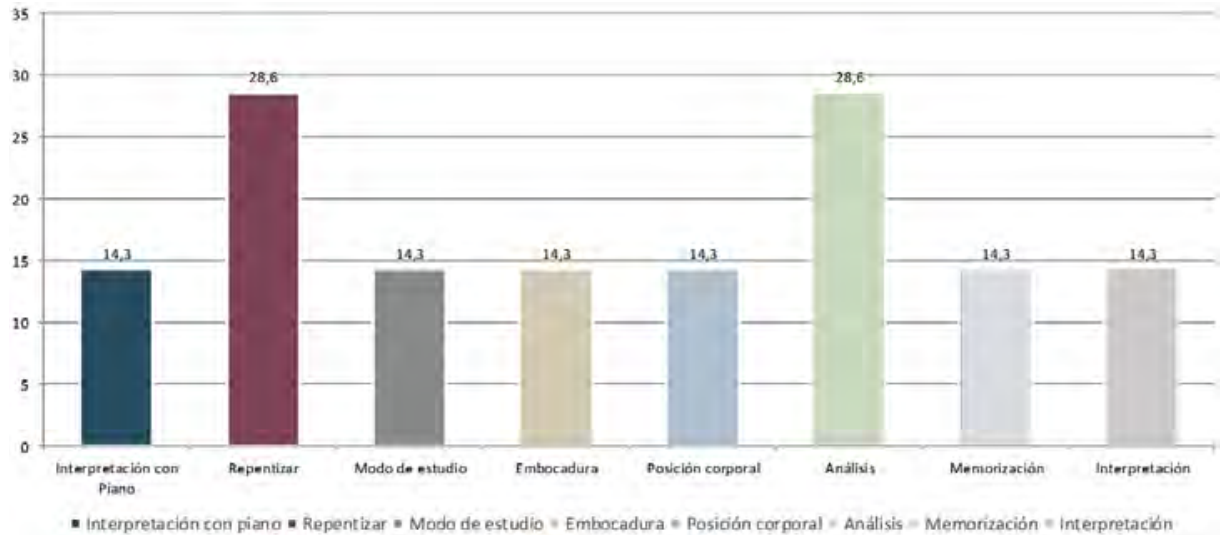

Fuente: elaboración propia.

Figura 5. Aspectos que precisarían mayor trabajo durante las EE.PP.

también señaló una serie de aspectos que trabajaría más durante las Enseñanzas Profesionales de Clarinete (figura 5).

Atendiendo a las necesidades de mejora señaladas por el alumnado y a las deficiencias formativas autodetectadas (figura 4), datos que fueron necesarios porque no se contaba con bibliografía relacionada, el alumnado de Clarinete que participó en la experiencia realizó un cuestionario previo (véase tabla vi) para conocer su grado de competencia en distintos elementos. Así, se obtuvieron los siguientes resultados previos (figura 6) y posteriores a la intervención (figura 7). 


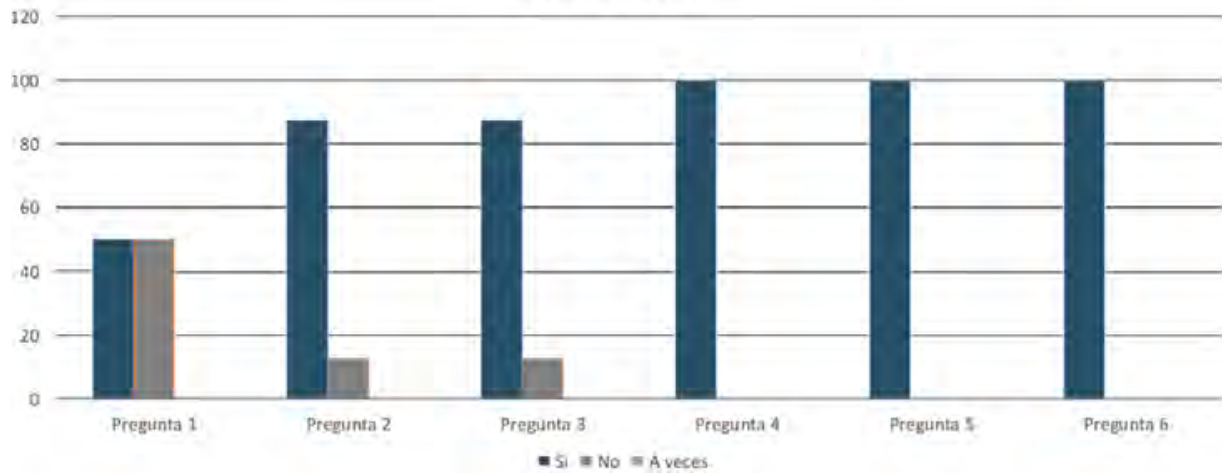

Fuente: elaboración propia.

Figura 6. Datos previos del alumnado participante.

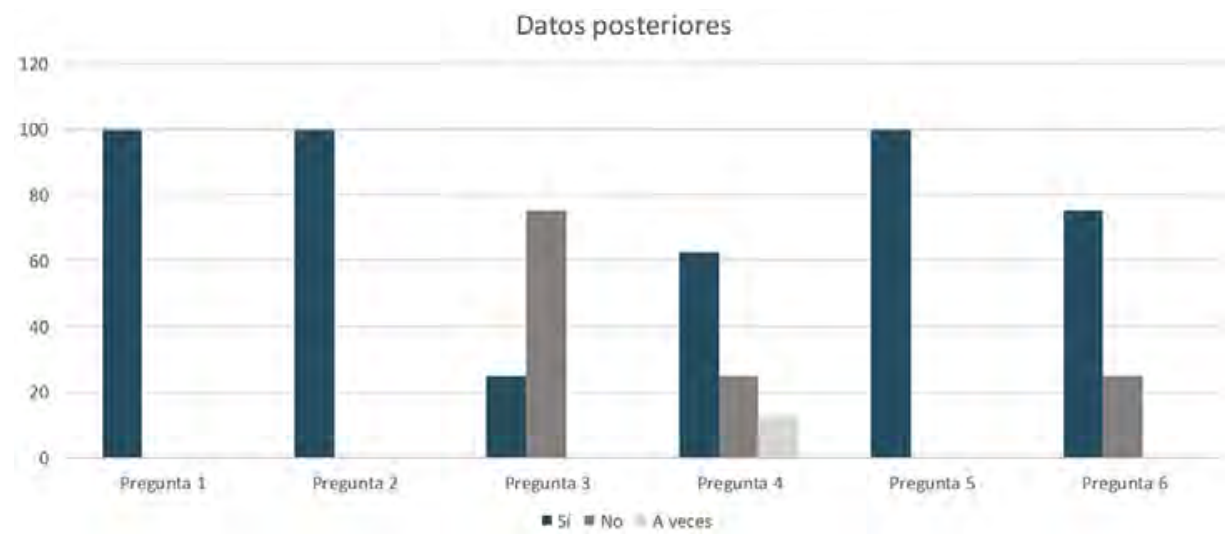

Fuente: elaboración propia.

Figura 7. Datos posteriores del alumnado participante.

Respecto a las cuestiones planteadas en la tabla vI, se obtuvieron los siguientes resultados: frente a un $50 \%$ inicial de respuestas positivas, al terminar la intervención el $100 \%$ del alumnado dio una respuesta positiva a la pregunta primera. Algo similar sucedió con la segunda, donde inicialmente un $87,5 \%$ respondió afirmativamente, frente a un $12,5 \%$ que contestó de forma negativa; aunque al finalizar el $100 \%$ respondió afirmativamente. En la tercera, se pasó del 87,5\% inicial que dijo que sí y el $12,5 \%$ que dijo que no, a un $25 \%$ afirmativo y un $75 \%$ negativo. Ya ante la cuarta, del $100 \%$ inicial que consideró una respuesta afirmativa, hubo un reparto entre el 
$62,5 \%$ del sí, el $25 \%$ del no y el $12,5 \%$ que consideraba que a veces. En la quinta pregunta no se variaron los resultados obtenidos: el 100\% del alumnado respondió afirmativamente antes y después de la intervención realizada. Y, finalmente, en la sexta pregunta sí se modificaron los resultados, ya que del 100\% inicial que contestó afirmativamente se pasó al 75\% que dijo que sí y el 25\% que no.

\section{DISCUSIÓN}

Las entrevistas realizadas al profesorado de la especialidad de Clarinete en los Conservatorios Profesionales de la Vega Baja y Murcia capital, y Superiores de Alicante y Murcia, mostraron que el alumnado afrontaba las pruebas con problemas a la hora de diferenciar estilos musicales, con problemas técnicos (muy básicos, en ocasiones), etc. El alumnado, por su parte, consideraba que uno de sus principales problemas a la hora de afrontar las pruebas, además de las dificultades a la hora de memorizar o de repentizar, era el miedo escénico.

Los datos recogidos antes, durante y tras la finalización de la experiencia, mostraron la necesidad de sistematizar el proceso de enseñanza/aprendizaje de forma que el estudiante pudiese contar con recursos para trabajar en casa y mejorar su interpretación cuando estuviese nuevamente en el aula. Se llegó a la conclusión de que el alumnado necesitaba ir más allá de la repetición con el criterio de su docente, porque ahora necesitaban explicaciones y no la típica consideración de que el músico nace, puesto que todo se puede aprender. El gusto, el carácter, el sentido de la Música durante la interpretación se aprenden porque son fruto de la reflexión en torno al análisis de los distintos elementos que conforman una partitura musical, aunque otra cosa es si se tiene mayor o menor "gusto" a la hora de la interpretación, pero eso ya es cuestión de opiniones. A los datos que se pretendió conseguir con estas entrevistas, se sumó una búsqueda bibliográfica en distintos repositorios digitales que mostró la inexistencia de referencias a la Didáctica instrumental en los Conservatorios de Música; no obstante, sí se encontraron referencias a la triste realidad sobre la carencia de las mismas: Jorquera (2002) se preguntaba si existía una didáctica del instrumento, porque el peso de la tradición de la imitación pesaba demasiado. En este sentido, las entrevistas realizadas al alumnado demostraron que necesitaban algo más para comprender la Música que repetir la interpretación del docente. Estudios de otros autores como Bautista y Pérez-Echeverría (2008) también han insistido en mostrar que lo que ellos llaman "teorías de la naturaleza directa e interpretativa» (la imitación del docente) deben ser sustituidas por el aprendizaje constructivista. Considerando todo esto, resultó lícito plantear unas pautas metodológicas que contribuyesen precisamente a construir el aprendizaje de la interpretación musical. Ahora bien, esta propuesta de pautas metodológicas enfocó ese estilo constructivista a las características de la prueba de acceso a Grado Superior, para que el estudiantado la pudiese superar exitosamente.

Los datos obtenidos del profesorado de Grado Superior que fue entrevistado mostraron que todos ellos consideraron no necesaria sino imprescindible una sistematización, que no sólo partiese del Tercer Ciclo, sino que se iniciase en las etapas 
anteriores. Todos estaban detectando gran cantidad de problemas de base cuya única explicación posible consideraron que era la falta de pautas para el estudiante a la hora de completar su estudio en casa. Por eso, el momento inicial propuesto para el trabajo del control respiratorio, postural y mental adquirió un peso tan importante en estas pautas metodológicas desarrolladas: se intentó dotar al estudiante de recursos y propuestas suficientes para que pudiese seleccionar para el estudio en casa las más convenientes a sus capacidades.

Las entrevistas al profesorado de Grado Superior y de EE.PP. mostraron que estaban preocupados por esas dificultades del alumnado a la hora de afrontar la interpretación de una obra, pero no dieron muestras de ahondar en la investigación didáctica en esta dirección. Esto vino a justificar la propuesta metodológica presentada en este artículo, ya que se trataba de ofrecer una respuesta didáctica a un problema formativo relacionado con el modelo de prueba de acceso vigente desde la reforma LOGSE, LOE, etc. Esta metodología que se propuso partió de las exigencias de ambas etapas, es decir, del currículo de EE.PP. y de las características de cada una de las partes de la prueba de acceso de Clarinete: repentización, interpretación con piano/solo (una obra de memoria) y analizar una obra (no de clarinete). En este sentido, los comentarios recogidos entre el alumnado se centraron en la importancia (y gratitud) que otorgaron al hecho de orientar las clases hacia la prueba de acceso.

Como profesionales de la docencia en Enseñanzas Elementales y Profesionales, se ha observado que en esta prueba existe un porcentaje alto de suspensos, debido a diferentes problemas relacionados con aspectos técnicos y con aspectos de índole más personal. Toda propuesta metodológica que se precie debe contribuir al proceso madurativo del estudiante, de modo que cuando se trabaje la interpretación de las obras, previamente debe haberse trabajado su análisis, que facilitará la maduración de la misma y, por tanto, una mejor interpretación. Aunque debe señalarse que el autocontrol corporal y del estado nervioso no sólo es fruto de la maduración personal, sino que desde el aula instrumental debe trabajarse para favorecerla, desde el ofrecimiento de distintas formas de trabajo de la técnica, de la columna de aire, de la postura... Sólo así el estudiante superará su miedo al escenario, derivado de la falta de control de alguno de los elementos citados, lo que le llevará a una interpretación madura y de calidad ante el tribunal de la prueba de Clarinete.

Aspectos tan negativos como los nervios y la tensión muscular que conlleva provocan problemas en el desarrollo de las obras de memoria ante el tribunal, tal como muestran los datos recogidos entre el alumnado. La falta de práctica de la memoria musical en el aula y, por tanto, la falta de recursos para continuar perfeccionándola en casa aumentan la intranquilidad del estudiante ante el examen de acceso. Destacar que la memoria musical necesita un trabajo en el aula y en el escenario: Jorquera (2002) indicó que la memoria no debía ser únicamente la del profesor, porque el alumnado debe personalizar y madurar la suya propia para afrontar una correcta interpretación memorística. Bautista y Pérez-Echeverría (2008) han propuesto pautas para aprender una partitura, que se tuvieron en cuenta porque se compartía la visión de que no sólo se trata de notas y técnica, sino que la interpretación musical pasa por aunar los conocimientos teórico-prácticos aprendidos con otras materias de las EE.PP. Ante esto, la metodología propuesta partió del trabajo en el aula de 
la memoria musical, ya que el alumnado mostraba su desacuerdo ante su falta de trabajo en el aula y, por consiguiente, ante la falta de herramientas para afrontar su trabajo fuera de ella.

La elaboración de la propuesta metodológica también tuvo en cuenta las consideraciones de autores como Palacios (1998), que proponía el trabajo del autocontrol por parte del estudiante y que éste aprendiese a detectar sus problemas y resolverlos sin ayuda; y de Torrado y Pozo (2008), que indicaban que había que ayudarlos a supervisar su propia práctica. No obstante, a pesar de que se tomó en consideración esa idea de construir el aprendizaje desde el propio estudiante, la propuesta desarrollada partió de una primera reconducción del proceso de enseñanza/aprendizaje en el aula para paliar los problemas cognitivos derivados de la larga tradición de la imitación en el aula del Conservatorio de Música.

Para enfocar el examen de Análisis musical, si bien el alumnado de Clarinete era formado por el profesorado específico en este sentido, las pautas trabajadas desde las obras de clarinete, les hicieron enfrentarse con mayor seguridad a esta prueba, según afirmó el alumnado participante en esta investigación. Habían trabajado en el aula de Clarinete el estilo de la obra, los puntos armónicos más importantes, la forma o estructura de la obra a nivel estilístico también. Esto contribuyó a aportar mayor madurez interpretativa al aprendizaje específico conseguido en el aula de Análisis de EE.PP. El "plus» de madurez del trabajo en el aula de Clarinete reportó mayor capacidad de razonamiento a la hora de responder a las preguntas que formaban parte del examen citado. Bautista y Pérez-Echeverría (2008) han comprobado en sus investigaciones que el hecho de trabajar el análisis del repertorio en el aula facilita la memorización del mismo; y, como se comentó anteriormente, puesto que la prueba de acceso exige una obra de memoria, si el repertorio es analizado en el aula, no sólo se estará facilitando dicha memorización, sino también una correcta interpretación del mismo. En esa misma línea de la importancia de las materias teóricas se movió esta propuesta metodológica, ya que sin esos conocimientos el alumnado no podría hacer frente a una interpretación con la suficiente rigurosidad histórico-estilística.

\section{CONCLUSIONES}

La legislación vigente para las enseñanzas obligatorias siempre ha hablado de un aprendizaje no sólo significativo para el estudiante, sino también constructivista. Desde esta perspectiva, en esta investigación se consideró que sólo defendiendo un proceso educativo significativo y constructivo se podría optimizar la interpretación del alumnado de cara a una prueba de acceso a Grado Superior. Y, tratando de seguir esa estela, se extrapolaron esas características de la pedagogía general al mundo de los Conservatorios de Música; al fin y al cabo, se quería dar respuesta a las necesidades detectadas en el alumnado participante en esta investigación. De manera que las pautas metodológicas desarrolladas con el estudiantado de Clarinete de Tercer Ciclo de EE.PP., que participó de esta experiencia investigadora, pretendieron ofrecer una sistematización de todo un corpus teórico-práctico que pudiese facilitar la labor del docente y del alumnado de los Conservatorios de Música. 
Una sistematización metodológica para el último ciclo de EE.PP. no es sólo una necesidad que permitirá dotar de mayor sentido académico y de rigurosidad a estas enseñanzas, sino que supone un proceso que se ajusta a las exigencias legislativas (y necesidades) de todos los miembros de la comunidad educativa.

Se puede concluir defendiendo lo necesario de investigar alrededor del proceso didáctico en el aula instrumental de los Conservatorios de Música. Así, la preparación para las pruebas de acceso a Grado Superior no supondrá un punto de inflexión tan negativo en la vida del estudiante de Música. Se ha venido a demostrar que sólo una formación desde el aprendizaje significativo para el estudiante de Clarinete y que implique un proceso constructivo de su propio aprendizaje le facilitará el correcto desarrollo de todo su potencial interpretativo, técnico y expresivo, que, al mismo tiempo, le ayudará a superar las pruebas de acceso de forma satisfactoria. 


\section{REFERENCIAS BIBLIOGRÁFICAS}

Bautista, A., Pérez-Echeverría, M. (2008). «¿Qué consideran los profesores de instrumento que deben enseñar en sus clases?». Cultura y Educación, 20 (1), 14-37.

Bernabé, M. y Torres, M.A. (2017). «¿Qué formación didáctica recibe el futuro profesorado de Clarinete en el Grado Superior? Reflexiones y propuesta curricular». ARBOR, 193 (783), 1-29.

Jorquera, M. ${ }^{a}$ C. (2002). «Existe una didáctica del instrumento musical?». Revista electrónica de LEEME, 9, 1-12.

Palacios, M. (2008). «La Didáctica aplicada a la enseñanza del instrumento». Revista Electrónica LEEME, 2, 1-7.

Torrado, J. y Pozo, J. (2008). «Metas y estrategias para una práctica constructiva en la enseńanza instrumental». Cultura y Educación, 20 (1), 35-48. 
